Research Article

\title{
Elevated Blood Urea Nitrogen-to-Serum Albumin Ratio as a Factor That Negatively Affects the Mortality of Patients with Hospital-Acquired Pneumonia
}

\author{
Ding-Yun Feng $\mathbb{D}^{1},{ }^{1}$ Yu-Qi Zhou, ${ }^{1}$ Xiao-Ling Zou, ${ }^{1}$ Mi Zhou, ${ }^{2}$ Hai-Ling Yang, \\ Xiao-Xia Chen, ${ }^{3}$ and Tian-Tuo Zhang $\mathbb{D}^{1}$ \\ ${ }^{1}$ Department of Respiratory and Critical Care Medicine, Third Affiliated Hospital of Sun Yat-Sen University, \\ Institute of Respiratory Diseases of Sun Yat-Sen University, Guangzhou, China \\ ${ }^{2}$ Department of Surgery Intensive Care Unit, Third Affiliated Hospital of Sun Yat-Sen University, Guangzhou, China \\ ${ }^{3}$ Department of Medical Record, Third Affiliated Hospital of Sun Yat-Sen University, Guangzhou, China
}

Correspondence should be addressed to Tian-Tuo Zhang; zhtituli@163.com

Received 20 January 2019; Revised 22 May 2019; Accepted 3 June 2019; Published 16 June 2019

Academic Editor: Massimo Girardis

Copyright (c) 2019 Ding-Yun Feng et al. This is an open access article distributed under the Creative Commons Attribution License, which permits unrestricted use, distribution, and reproduction in any medium, provided the original work is properly cited.

\begin{abstract}
This study aimed to evaluate the factors that affect 30-day mortality of patients with HAP. The data used in this study were collected from all HAP occurred in our hospital between January 2014 and December 2017. A total of 1158 cases were included. $150(13.0 \%)$ of whom died within 30 days. This reported mortality identified by the univariate Cox regression analysis is found to have been affected by the following factors: age greater than 70 years, presence of diabetes mellitus and chronic obstructive pulmonary disease, gastric tube intubation, administration of proton-pump inhibitor, blood albumin level less than $30 \mathrm{~g} / \mathrm{l}$, elevated neutrophil-to-lymphocyte ratio, antibiotics therapy in the preceding 90 days, intensive care unit (ICU) admission, blood lymphocyte count less than $0.8 \times 10^{9} / \mathrm{L}$, elevated blood urea nitrogen/albumin (BUN/ALB) level, and presence of multidrugresistant (MDR) pathogens. In the second multivariate analysis, administration of proton-pump inhibitor, administration of antibiotics in the preceding 90 days, ICU admission, blood lymphocyte count less than $0.8 \times 10^{9} / \mathrm{L}$, elevated BUN/ALB level, and presence of MDR pathogens were still associated with 30-day mortality. The area under the receiver operating characteristic curves in the BUN/ALB predicting 30-day mortality due to HAP was 0.685. A high BUN/ALB was significantly associated with a worse survival than a low BUN/ALB $(P<0.001)$. Therefore, an elevated BUN/ALB level is a risk factor for mortality on patients with HAP.
\end{abstract}

\section{Introduction}

Nosocomial or hospital-acquired pneumonia (HAP) is a common infection associated with a prolonged hospital length of stay and high patient mortality, generally with symptom onset at $48 \mathrm{~h}$ or more after admission to the hospital, or within 14 days of discharge from the hospital [1]. In the United States, it is the second most common nosocomial infection, after urinary tract infections, with the incidence rate ranging from $0.5 \%$ to $1 \%$ [2]. HAP is also common in China with an incidence rate of $1.74 \%-6.4 \%$ $[3,4]$, as well as in Vietnam where it is the most common nosocomial infection in adult intensive care units (ICUs) $[5,6]$. HAP is a common hospital-acquired infection with a prolonged hospital length of stay and high patient mortality [1]. HAP is associated with a mortality rate ranging from $20 \%$ to $60 \%$, especially in the ICU [7-9]. The attributable mortality is probably higher in some specific populations such as patients with chronic obstructive pulmonary disease (COPD) [10] and psychiatric diseases [11]. Hence, it is important to assess the factors related to HAP to predict patient prognosis. Therefore, this study aimed to evaluate the factors that affect 30-day mortality of patients with HAP, in order to improve its prognosis in the future. 


\section{Materials and Methods}

Data related to all incidence of HAP that occurred in the third affiliated hospital of Sun Yat-sen University, Guangdong, China, between January 2014 and December 2017 were collected. Variables of interest included age, gender, comorbidity, central venous catheter use, prior treatment, laboratory data, and 30-day mortality. All patients included were aged at least 18 years. The criteria for a diagnosis of HAP [2] included a new pulmonary infiltrate (occurring $\geq 48 \mathrm{~h}$ after admission) associated with at least one of the following: new or increased cough with or without purulent tracheobronchial secretion or new pathogenic bacteria isolated from sputum or tracheal aspirate culture with $\geq 10^{4}$ colony-forming units $/ \mathrm{ml}$, fever $\left(>37.8^{\circ} \mathrm{C}\right)$ or hypothermia $\left(<35.6^{\circ} \mathrm{C}\right)$, leukocytosis, left shift, or leucopenia based on local normal values. Cases of ventilator-associated pneumonia and acquired immunodeficiency syndrome and those with missing key data were excluded.

Pathogenic bacteria isolated from the clinical specimens were further characterized by conventional biochemical tests to identify the specific strain by using standard microbiologic methods [12]. Pathogenic organism susceptibility testing was conducted using the microdilution method (MicroScan System; Baxter Healthcare, West Sacramento, CA, USA), and the results were interpreted using the National Committee for Clinical Laboratory Standards guidelines published in 2012 (Clinical \& Laboratory Standards Institute, 2012). Here, multidrug-resistant (MDR) pathogens were defined as organisms resistant to at least one agent of three or more antimicrobial categories in susceptibility tests of isolates from patients with HAP [13].

2.1. Statistical Analysis. The 30-day survival was the main endpoint of this study. Survival was defined as the time interval between HAP diagnosis and death or the last followup. Multivariate analysis using a stepwise Cox proportional hazards model was used to test for independent significance of baseline characteristics and explanatory variables. The performance of relevant parameters was assessed by using the Kaplan-Meier method, and differences in survival between groups were compared by using the log-rank test. In addition, the receiver operating characteristic (ROC) curves of BUN/ALB for predicting 30-day mortality with HAP were plotted. Sensitivity of a score less than the cutoff point indicated 30-day mortality, while specificity of that greater than the cutoff point indicated survival beyond 30 days, both of which could be evaluated for each possible cutoff point. The cutoff point representing the highest Youden index (specificity + specificity - 1) was selected as the optimal threshold value. Hazard ratios (HRs) and 95\% confidence intervals (CIs) were pooled to measure the effects of relevant parameters on prognosis. An HR greater than 1 indicated a worse prognosis in patients with a relevant parameter, while a HR less than 1 indicated a better prognosis. The criterion for statistical significance was set at an $\alpha$ of 0.05 , and all $P$ values were based on two-sided tests. Statistical analysis was performed using IBM SPSS Statistics, version 20 (IBM Corp, Armonk, NY, USA).

\section{Results}

Among the 210,417 inpatients, $1472(0.7 \%)$ cases of HAP were recorded. 314 of which were excluded because of missing key data or presence of repeated infection; finally, only a total of 1158 cases were included (Figure 1 and Table 1). Within 30 days, 150 (13.0\%) patients died and 566 (48.9\%) patients received antibiotic therapy during the last 90 days, while $30(2.6 \%)$ patients were admitted in the ICU. A total of $327(28.2 \%)$ patients had a blood lymphocyte count less than $0.8 \times 10^{9} / \mathrm{L}$ and blood urea nitrogen/albumin (BUN/ALB) level of $0.21 \pm 0.17$, and 193 (16.7\%) patients had MDR pathogens in their system (Table 2).

The univariate Cox regression analysis identified the following independent factors that affect 30-day mortality with HAP (Table 3): age greater than 70 years, presence of diabetes mellitus and COPD, gastric tube intubation, administration of proton-pump inhibitor, blood albumin level less than $30 \mathrm{~g} / \mathrm{l}$, elevated neutrophil-to-lymphocyte ratio, antibiotics therapy in the preceding 90 days, ICU admission, blood lymphocyte count less than $0.8 \times 10^{9} / \mathrm{L}$, elevated BUN/ ALB levels, and presence of MDR pathogens. Multivariable analysis revealed that administration of proton-pump inhibitor $(\mathrm{OR}=1.508 ; 95 \% \mathrm{CI}, 1.003-2.268 ; P=0.048)$, antibiotic therapy in the preceding 90 days $(\mathrm{OR}=1.875 ; 95 \%$ CI, 1.278-2.751; $P=0.001)$, ICU admission ( $\mathrm{OR}=2.405$; 95\% CI, 1.334-4.338; $P=0.004$ ), blood lymphocyte count less than $0.8 \times 10^{9} / \mathrm{L}(\mathrm{OR}=1.626 ; 95 \% \mathrm{CI}, 1.126-2.348$; $P=0.009)$, elevated BUN/ALB $(\mathrm{OR}=3.871 ; 95 \%$ CI, 2.174-6.893; $P<0.001)$ levels, and presence of MDR pathogens $(\mathrm{OR}=1.870 ; 95 \% \mathrm{CI}, 1.252-2.794 ; P=0.002)$ were still associated with 30 -day mortality. ROC analysis presented similar results with Cox regression and revealed that the BUN/ALB was a significant predictor of 30-day survival. The area under the ROC curves in the BUN/ALB predicting 30-day mortality with HAP was 0.685 (Figure 2), and the cutoff point was 0.165 with the highest predictive performance for both specificity and sensitivity (Figure 2). A high BUN/ALB level was significantly associated with worse survival than that of a low BUN/ALB $(P<0.001)$ (Figure 3 ).

\section{Discussion}

We revealed that the mean mortality rate in this study was $13.0 \%$, consistent with the HAP mortality reported by other researchers $[8,14]$. In the ICUs, the mortality was the highest, similar to that seen in the previous studies $[8,15]$ because the patients often had multiple organ dysfunction or underwent bronchoscopy [15]. Furthermore, we identified significant prognostic factors for 30-day mortality among HAP patients, including administration of proton-pump inhibitor, antibiotic therapy in the preceding 90 days, ICU admission, blood lymphocyte count less than $0.8 \times 10^{9} / \mathrm{L}$, elevated BUN/ALB levels, and presence of MDR pathogens.

As HAP is often associated with peptic disease and nonsteroidal drugs are usually used to allay patients' fever, 


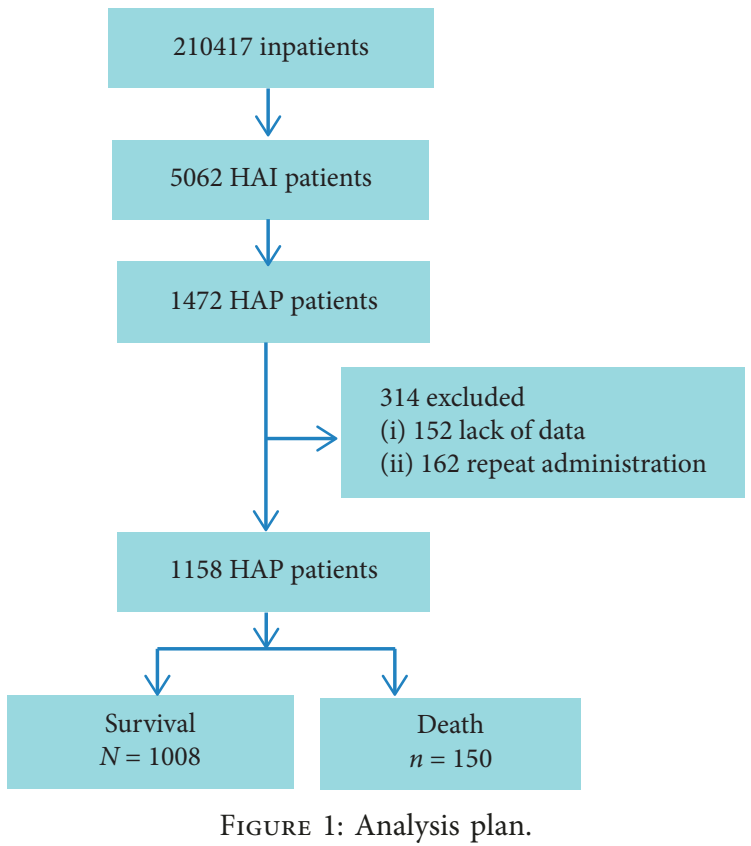

TABLE 1: Epidemiology and 30-day mortality of HAP in each department.

\begin{tabular}{|c|c|c|c|c|}
\hline Department & Inpatient, $n=210417(\%)$ & HAP, $n=1158(\%)$ & Death, $n=150(\%)$ & 30-day mortality (\%) \\
\hline SICU & $465(0.22)$ & $7(0.60)$ & $4(2.7)$ & 57.1 \\
\hline MICU & $957(0.45)$ & $23(1.99)$ & $11(7.3)$ & 47.8 \\
\hline Gastroenterology & $6366(3.03)$ & $22(1.90)$ & $9(6.0)$ & 40.9 \\
\hline Rheumatology & $6479(3.08)$ & $23(1.97)$ & $8(5.3)$ & 34.8 \\
\hline Oncology & $11706(5.56)$ & $79(6.82)$ & $25(16.7)$ & 31.6 \\
\hline Traditional Chinese medicine & $3652(1.74)$ & $7(0.60)$ & $2(1.3)$ & 28.6 \\
\hline Respiratory medicine & $5294(2.51)$ & $45(3.87)$ & $11(7.3)$ & 24.4 \\
\hline Infectious diseases & $19651(9.33)$ & $99(8.55)$ & $20(13.3)$ & 20.2 \\
\hline Nephrology & $4308(2.04)$ & $39(3.37)$ & $7(4.7)$ & 17.9 \\
\hline Gastrointestinal surgery & $8326(3.96)$ & $42(3.63)$ & $6(4.0)$ & 14.3 \\
\hline Cardiovascular & $9623(4.57)$ & $141(12.18)$ & $18(12)$ & 12.8 \\
\hline Invasive technology & $8348(3.97)$ & $9(0.78)$ & $1(0.7)$ & 11.1 \\
\hline Spine surgery & $3580(1.70)$ & $21(1.81)$ & $2(1.3)$ & 9.5 \\
\hline Neurology & $12405(5.90)$ & $144(12.44)$ & $12(8.0)$ & 8.3 \\
\hline Endocrinology & $9307(4.42)$ & $26(10.79)$ & $2(1.3)$ & 7.7 \\
\hline Neurosurgery & $2886(1.37)$ & $57(4.92)$ & $4(2.7)$ & 7 \\
\hline Rehabilitation & $4171(1.98)$ & $125(10.79)$ & $5(3.3)$ & 4 \\
\hline Hepatological surgery & $12860(6.11)$ & $69(5.96)$ & $2(1.3)$ & 2.9 \\
\hline Psychiatry & $11668(5.55)$ & $126(10.88)$ & $1(0.7)$ & 0.8 \\
\hline Joint surgery & $5124(2.44)$ & $9(0.78)$ & 0 & 0 \\
\hline Urology & $10853(5.16)$ & $17(1.47)$ & 0 & 0 \\
\hline Thyroid surgery & $10766(5.12)$ & $13(1.12)$ & 0 & 0 \\
\hline ENT & $10636(5.05)$ & $8(0.69)$ & 0 & 0 \\
\hline Dermatology & $2979(1.42)$ & $1(0.09)$ & 0 & 0 \\
\hline Gynaecology and obstetrics & $28007(13.31)$ & $6(0.52)$ & 0 & 0 \\
\hline
\end{tabular}

HAP: hospital-acquired pneumonia; SICU: surgical intensive care unit; MICU: medical intensive care unit; ENT: ear, nose, and throat.

the administration of proton-pump inhibitor was common in HAP patients. In the current study, we found that the administration of proton-pump inhibitor had a negative effect on HAP patients' 30-day mortality, consistent with the literature report [16]. Whether patients received antibiotic therapy in the preceding 90 days showed a significant difference in the outcomes of the present study, suggesting its importance as a prognostic factor for 30-day mortality with
HAP. This finding is similar to that of previous studies that suggested the impact of virulence and antibiotic tolerance [17]. Meanwhile, antibiotic therapy in the preceding 90 days resulted in an increase of MDR pathogenesis, thereby leading to worse prognosis and higher mortality [18]. Presence of MDR pathogens had a negative effect on HAP patients' 30 -day mortality in this study. It was similar to this study because MDR pathogens' virulence was stronger and 
TABLe 2: Demographic, laboratory, and clinical variables of HAP.

\begin{tabular}{lr}
\hline Characteristics & Value \\
\hline Age $>70$ years & $409(35.3 \%)$ \\
Gender: male & $723(62.4 \%)$ \\
Smoke & $248(21.4 \%)$ \\
Diabetes mellitus & $230(19.9 \%)$ \\
COPD & $55(4.7 \%)$ \\
Antibiotics therapy in the preceding 90 days & $566(48.9 \%)$ \\
Stomach tube intubation & $307(26.5 \%)$ \\
Central venous catheterization & $197(17.0 \%)$ \\
Use of PPI & $745(64.3 \%)$ \\
ICU admission & $30(2.6 \%)$ \\
ALB $<30$ g/L & $124(10.7 \%)$ \\
WBC, $\times 10^{9} / \mathrm{L}$ & $10.44 \pm 5.72$ \\
Lymphocyte count $<0.8 * 10^{9} / \mathrm{L}$ & $327(28.2 \%)$ \\
NLR & $9.08 \pm 9.06$ \\
BUN/ALB & $0.21 \pm 0.17$ \\
MDR pathogens & $193(16.7 \%)$ \\
Related mortality & $150(13.0 \%)$ \\
\hline
\end{tabular}

HAP: hospital-acquired pneumonia; COPD: chronic obstructive pulmonary disease; use of PPI: use of proton-pump inhibitor; ICU admission: intensive care unit admission; ALB: albumin; WBC: white blood cell; NLR: neutrophil-to-lymphocyte count ratio; BUN/ALB: blood urea nitrogen/blood albumin; MDR: multidrug resistant.

TABLE 3: Univariate and multivariate cox regression analyses of factors affecting 30-day mortality with HAP.

\begin{tabular}{|c|c|c|c|c|c|c|c|c|}
\hline \multirow{2}{*}{ Characteristics } & \multirow{2}{*}{$\begin{array}{c}\text { Related mortality, } \\
n=150(13.0 \%)\end{array}$} & \multirow{2}{*}{$\begin{array}{c}\text { Survival, } n=1008 \\
(87.0 \%)\end{array}$} & \multicolumn{3}{|c|}{ Univariate } & \multicolumn{3}{|c|}{ Multivariate } \\
\hline & & & HR & $95 \% \mathrm{Cl}$ & $P$ & HR & $95 \% \mathrm{Cl}$ & $P$ \\
\hline Age $>70$ years & $71(47.3 \%)$ & $338(32.5 \%)$ & 1.701 & $1.234-2.343$ & 0.001 & 1.329 & $0.943-1.874$ & 0.105 \\
\hline Gender: male & $103(68.7 \%)$ & $620(61.5 \%)$ & 1.344 & $0.952-1.898$ & 0.093 & & & \\
\hline Smoke & $33(22.0 \%)$ & $215(21.3 \%)$ & 1.045 & $0.710-1.537$ & 0.825 & & & \\
\hline Diabetes mellitus & $40(26.7 \%)$ & $190(18.8 \%)$ & 1.524 & $1.061-2.188$ & 0.023 & 1.366 & $0.936-1.995$ & 0.106 \\
\hline COPD & $12(8.0 \%)$ & $43(0.43 \%)$ & 1.843 & $1.022-3.324$ & 0.042 & 1.075 & $0.569-2.030$ & 0.824 \\
\hline $\begin{array}{l}\text { Antibiotics therapy in the } \\
\text { preceding } 90 \text { days }\end{array}$ & $106(70.7 \%)$ & $460(45.6 \%)$ & 2.684 & $1.888-3.815$ & $<0.001$ & 1.875 & $1.278-2.751$ & 0.001 \\
\hline Stomach tube intubation & $54(36.0 \%)$ & $253(25.1 \%)$ & 1.606 & $1.151-2.442$ & 0.005 & 0.899 & $0.616-1.313$ & 0.582 \\
\hline Central venous catheterization & $32(21.3 \%)$ & $165(16.4 \%)$ & 1.348 & $0.912-1.992$ & 0.134 & & & \\
\hline Use of PPI & $118(78.7 \%)$ & $627(62.2 \%)$ & 2.138 & $1.447-3.161$ & $<0.001$ & 1.508 & $1.003-2.268$ & 0.048 \\
\hline ICU admission & $15(10.0 \%)$ & $15(1.5 \%)$ & 5.190 & $3.043-8.852$ & $<0.001$ & 2.405 & $1.334-4.338$ & 0.004 \\
\hline $\mathrm{ALB}<30 \mathrm{~g} / 1$ & $27(18.0 \%)$ & $97(9.6 \%)$ & 1.904 & $1.255-2.888$ & 0.002 & 1.346 & $0.874-2.074$ & 0.177 \\
\hline $\mathrm{WBC}$, mean $\pm \mathrm{SD},{ }^{*} 10^{9} / \mathrm{L}$ & $10.17 \pm 8.43$ & $10.47 \pm 5.19$ & 0.991 & $0.961-1.023$ & 0.586 & & & \\
\hline Lymphocyte count $<0.8 \times 10^{9} / 1$ & $64(42.7 \%)$ & $263(26.1 \%)$ & 1.964 & $1.421-2.714$ & $<0.001$ & 1.626 & $1.126-2.348$ & 0.009 \\
\hline NLR & $11.18 \pm 11.37$ & $8.76 \pm 8.62$ & 1.019 & $1.007-1.031$ & 0.002 & 1.004 & $0.987-1.022$ & 0.619 \\
\hline BUN/ALB & $0.33 \pm 0.28$ & $0.20 \pm 0.15$ & 9.100 & $5.647-14.663$ & $<0.001$ & 3.871 & $2.174-6.893$ & $<0.001$ \\
\hline MDR pathogens & $51(34.0 \%)$ & $142(14.1 \%)$ & 2.835 & $2.022-3.975$ & $<0.001$ & 1.870 & $1.252-2.794$ & 0.002 \\
\hline
\end{tabular}

HAP: hospital-acquired pneumonia; COPD: chronic obstructive pulmonary disease; use of PPI: use of proton-pump inhibitor; ICU admission: intensive care unit admission; ALB: albumin; WBC: white blood cell; NLR: neutrophil-to-lymphocyte count ratio; BUN/ALB: blood urea nitrogen/blood albumin; MDR: multidrug resistant.

the selection of antimicrobials was fewer [19]. In the current study, the 30-day mortality was still related to ICU admission because the illness maybe severe, implying the need for ICU admission [15], and antimicrobial resistance is an increasing concern in the ICUs worldwide [20]. Blood routine examination is common for patients, and the lymphocyte count is easy to calculate and very suitable for clinical application. Blood lymphocyte count less than $0.8 \times 10^{9} / \mathrm{L}$ was also an independent risk factor for 30 -day mortality in this study. It was similar to a previous study [21]. The BUN/ALB level is a simple but an independent predictor of mortality and severity of pneumonia [22]. An elevated BUN/ALB level is associated with higher 30-day mortality in the present study. Patients with pneumonia often had hydration status resulting in increasing reabsorption of urea by the kidneys, and elevation of BUN level is frequently observed [23]. It is interesting to note that earlier studies mostly focused on community-acquired pneumonia showed that nonsurvivors have significantly elevated BUN levels and lower serum albumin levels than those of survivors [24]. Our current study was one of the few researches highlighting BUN/ALB level as a predictor of 30-day mortality in a large sample. In addition, the cutoff point of BUN/ALB level was 0.165. A high BUN/ALB level was 


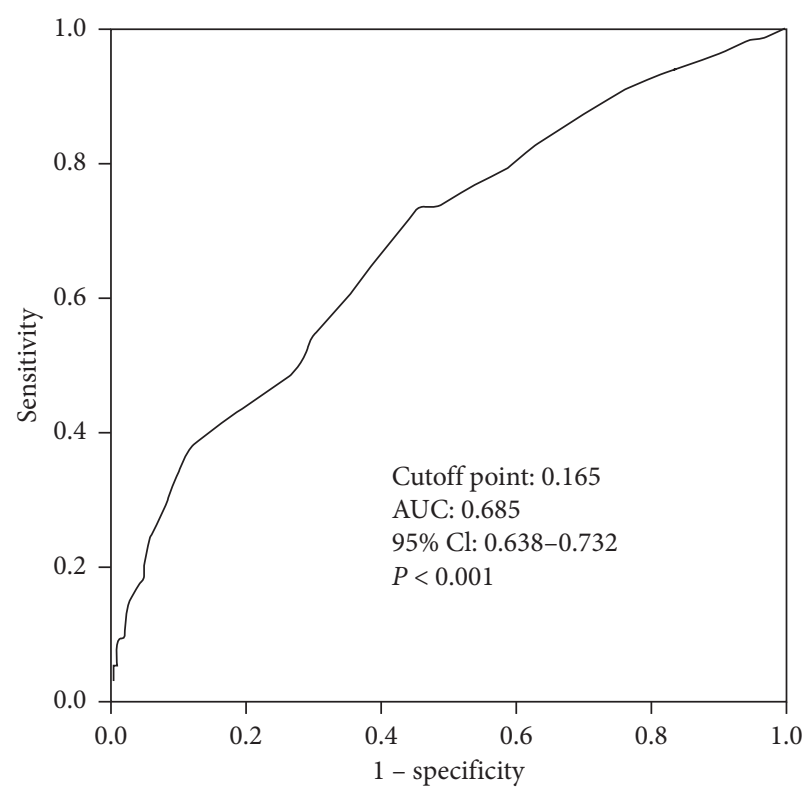

FIGURE 2: The ROC curve analysis of the BUN/ALB for predicting 30-day mortality with HAP. Abbreviation: ROC, receiver operating characteristic; AUC, area under the curve; $\mathrm{CI}$, confidence interval; BUN/ALB: blood urea nitrogen/blood albumin; HAP: hospitalacquired pneumonia.

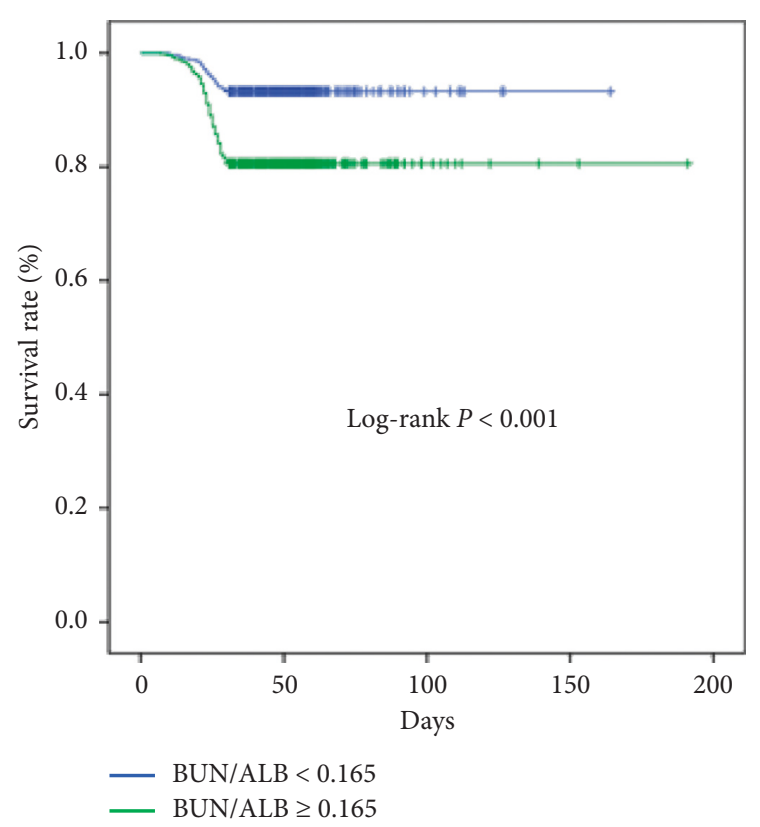

FIGURE 3: Kaplan-Meier curves of 30-day survival in HAP patients stratified by BUN/ALB. A high BUN/ALB was significantly associated with a worse survival than a low BUN/ALB $(P<0.001)$. HAP: hospital-acquired pneumonia; BUN/ALB: blood urea nitrogen/ blood albumin.

significantly associated with a worse survival than a low BUN/ALB. This will help clinicians to understand better what high BUN/ALB ratio means.

There are several limitations of our study that may decrease the impact of the results presented. First, as a single-center retrospective study, the assessment of HAP may contain bias. Second, there was no microbiologic documentation in half of the patients. It can be argued that some patients without etiologic diagnosis may not actually have had HAP. However, this is the usual situation in clinical practice, and our rate of etiologic diagnosis was similar to the ancillary reports in this field.

\section{Conclusion}

The purpose of the current study is to evaluate the factors that affect 30-day mortality of patients with HAP which have been highlighted in the findings. The results indicate that the mortality rates of HAP were different in each department and that peripheral blood BUN/ALB level is a simple but independent predictor of 30-day mortality with HAP. With solid statistics, the findings of this research are consistent with those of similar studies of prognostic factors of 30-day mortality with HAP.

\section{Data Availability}

The data used to support the findings of this study are available from the corresponding author upon request.

\section{Ethical Approval}

The study has been approved by the institutional review board of the hospital and the ethics committee of the Third Affiliated Hospital of Sun Yat-sen University.

\section{Conflicts of Interest}

The authors have no conflicts of interest to declare.

\section{Authors' Contributions}

Ding-Yun Feng and Yu-Qi Zhou contributed equally to this work.

\section{Acknowledgments}

We thank Dr. He-Liang Li, Jia-Sheng He, and Yi-Xiong Yuan for their help in data collection for this study.

\section{References}

[1] D. E. Craven and A. Chroneou, "Nosocomial pneumonia," in Principles and Practice of Infectious Diseases, G. L. Mandell, Ed., pp. 3717-3725, Elsevier Churchill Livingstone, Philadelphia, PA, USA, 7th edition, 2010.

[2] American Thoracic Society; Infectious Diseases Society of America, "Guidelines for the management of adults with hospital-acquired, ventilator-associated, and healthcareassociated pneumonia," American Journal of Respiratory and Critical Care, vol. 171, no. 4, pp. 388-416, 2005.

[3] X.-B. Tao, L.-H. Qian, Y. Li et al., "Hospital-acquired infection rate in a tertiary care teaching hospital in China: a crosssectional survey involving 2434 inpatients," International Journal of Infectious Diseases, vol. 27, pp. 7-9, 2014.

[4] J. Zhu, X. Zhang, G. Shi, K. Yi, and X. Tan, "Atrial fibrillation is an independent risk factor for hospital-acquired pneumonia," PLoS One, vol. 10, no. 7, Article ID e0131782, 2015. 
[5] V. D. Phu, H. F. L. Wertheim, M. Larsson et al., "Burden of hospital acquired infections and antimicrobial use in Vietnamese adult intensive care units," PLoS One, vol. 11, no. 1, Article ID e0147544, 2016.

[6] C. Ustun, S. Hosoglu, M. F. Geyik, Z. Parlak, and C. Ayaz, "The accuracy and validity of a weekly point-prevalence survey for evaluating the trend of hospital-acquired infections in a university hospital in Turkey," International Journal of Infectious Diseases, vol. 15, no. 10, pp. e684-e687, 2011.

[7] W. G. Melsen, M. M. Rovers, R. H. Groenwold et al., “Attributable mortality of ventilator-associated pneumonia: a meta-analysis of individual patient data from randomised prevention studies," The Lancet Infectious Diseases, vol. 13, no. 8, pp. 665-671, 2013.

[8] T. Kim, Y. P. Chong, S. Y. Park et al., "Risk factors for hospital-acquired pneumonia caused by carbapenemresistant gram-negative bacteria in critically ill patients: a multicenter study in Korea," Diagnostic Microbiology and Infectious Disease, vol. 78, no. 4, pp. 457-461, 2014.

[9] R. Bhadade, M. Harde, R. deSouza, A. More, and R. Bharmal, "Emerging trends of nosocomial pneumonia in intensive care unit of a tertiary care public teaching hospital in Western India," Annals of African Medicine, vol. 16, no. 3, pp. 107-113, 2017.

[10] D. Koulenti, E. Tsigou, and J. Rello, "Nosocomial pneumonia in 27 ICUs in Europe: perspectives from the EU-VAP/CAP study," European Journal of Clinical Microbiology \& Infectious Diseases, vol. 36, no. 11, pp. 1999-2006, 2017.

[11] T. Haga, K. Ito, K. Sakashita, M. Iguchi, M. Ono, and K. Tatsumi, "Risk factors for death from psychiatric hospitalacquired pneumonia," Internal Medicine, vol. 57, no. 17, pp. 2473-2478, 2018.

[12] K. C. Carroll and G. Funke, "Bacteriology," in Manual of Clinical Microbiology, J. Versalovic, K. C. Carroll, G. Funke, J. H. Jorgensen, M. L. Landry, and D. W. Warnock, Eds., pp. 15-51, American Society of Microbiology, Washington, DC, USA, 2011.

[13] A.-P. Magiorakos, A. Srinivasan, R. B. Carey et al., "Multidrug-resistant, extensively drug-resistant and pandrugresistant bacteria: an international expert proposal for interim standard definitions for acquired resistance," Clinical Microbiology and Infection, vol. 18, no. 3, pp. 268-281, 2012.

[14] F. Dehghan, N. Zolghadri, V. Boostani, A. Shafii, and T. E. Eftekhaari, "Resistance of gram negative bacteria in hospital acquired pneumonia: a prospective study," Brazilian Journal of Infectious Diseases, vol. 20, no. 1, pp. 113-114, 2016.

[15] R. Uvizl, M. Kolar, T. Herkel, M. Vobrova, and K. Langova, "Possibilities for modifying risk factors for the development of hospital-acquired pneumonia in intensive care patients: results of a retrospective, observational study," Biomedical Papers, vol. 161, no. 3, pp. 303-309, 2017.

[16] K. Hamai, H. Iwamoto, S. Ohshimo et al., "Use of proton pump inhibitors is associated with increased mortality due to nosocomial pneumonia in bedridden patients receiving tube feeding," Geriatrics \& Gerontology International, vol. 18, no. 8, pp. 1215-1218, 2018.

[17] T. Herkel, R. Uvizl, L. Doubravska et al., "Epidemiology of hospital-acquired pneumonia: results of a Central European multicenter, prospective, observational study compared with data from the European region," Biomedical Papers, vol. 160, no. 3, pp. 448-455, 2016.

[18] V. I. Enne, Y. Personne, L. Grgic, V. Gant, and A. Zumla, "Aetiology of hospital-acquired pneumonia and trends in antimicrobial resistance," Current Opinion in Pulmonary Medicine, vol. 20, no. 3, pp. 252-258, 2014.

[19] S. Gandra, K. K. Tseng, A. Arora et al., "The mortality burden of multidrug-resistant pathogens in India: a retrospective, observational study," Clinical Infectious Diseases, 2018.

[20] J. Cohen, "Confronting the threat of multidrug-resistant gram-negative bacteria in critically ill patients," Journal of Antimicrobial Chemotherapy, vol. 68, no. 3, pp. 490-491, 2013.

[21] L. A. Van Vught, B. P. Scicluna, M. A. Wiewel et al., "Comparative analysis of the host response to communityacquired and hospital-acquired pneumonia in critically ill patients," American Journal of Respiratory and Critical Care, vol. 194, no. 11, pp. 1366-1374, 2016.

[22] M. Ugajin, K. Yamaki, N. Iwamura, T. Yagi, and T. Asano, "Blood urea nitrogen to serum albumin ratio independently predicts mortality and severity of community-acquired pneumonia," International Journal of General Medicine, vol. 5, pp. 583-589, 2012.

[23] E. Woodford-Williams, "Respiratory tract disease: diagnosis and management of pneumonia in the aged," BMJ, vol. 1, no. 5485, pp. 467-470, 1966.

[24] J. H. Lee, J. Kim, K. Kim et al., "Albumin and C-reactive protein have prognostic significance in patients with community-acquired pneumonia," Journal of Critical Care, vol. 26, no. 3, pp. 287-294, 2011. 


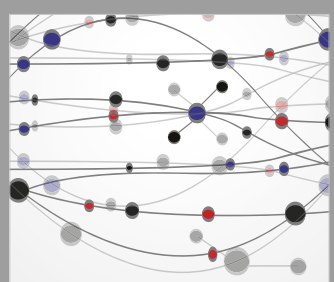

The Scientific World Journal
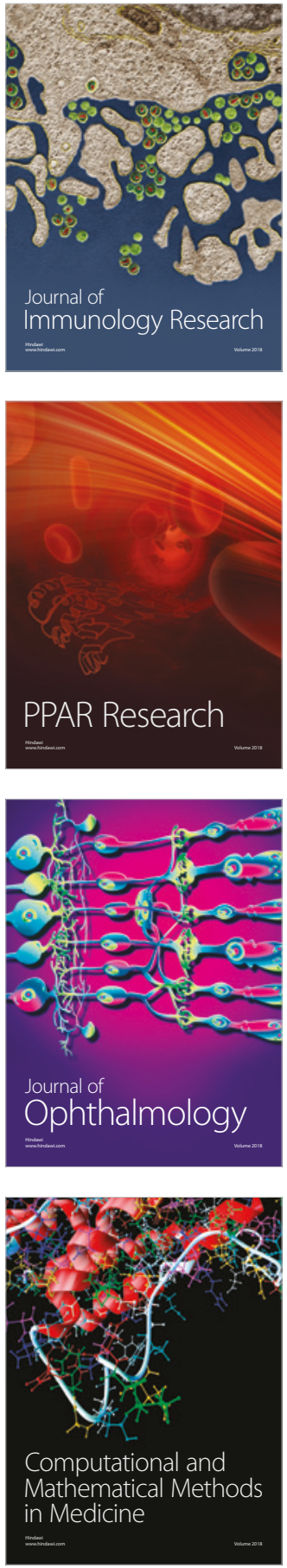

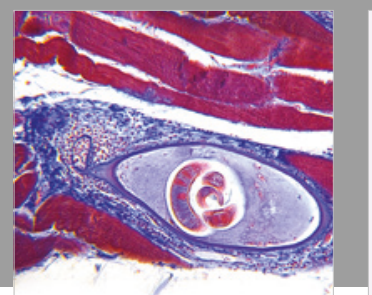

Gastroenterology Research and Practice

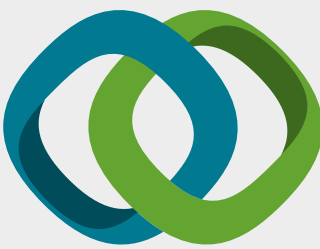

\section{Hindawi}

Submit your manuscripts at

www.hindawi.com
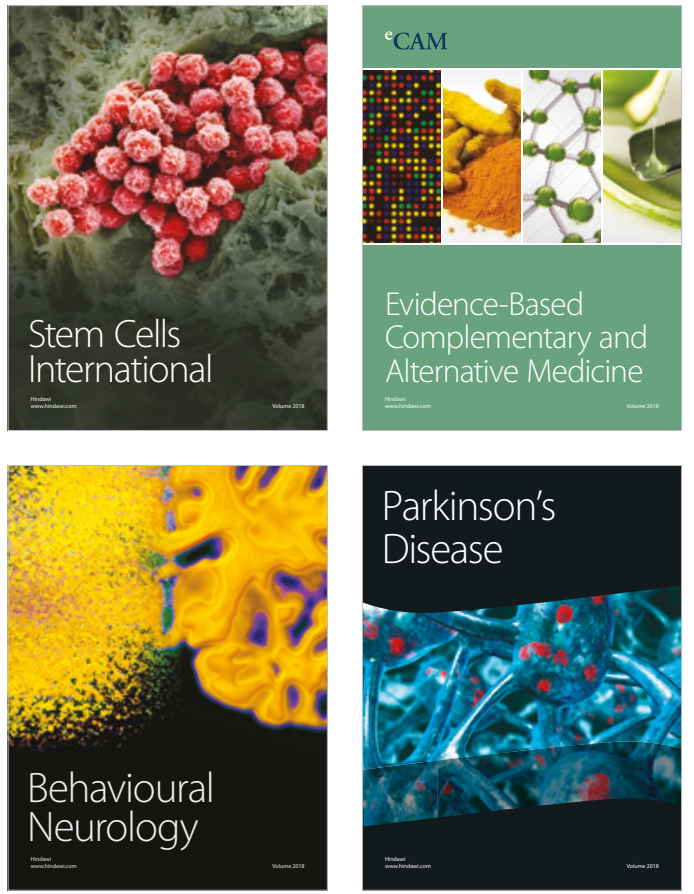

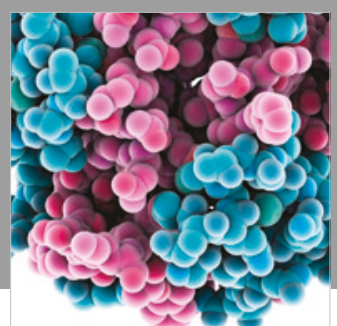

ournal of

Diabetes Research

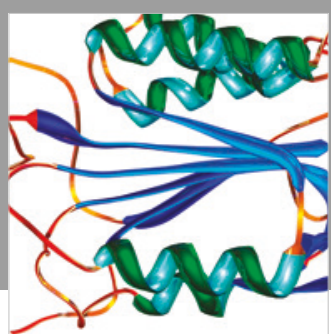

Disease Markers
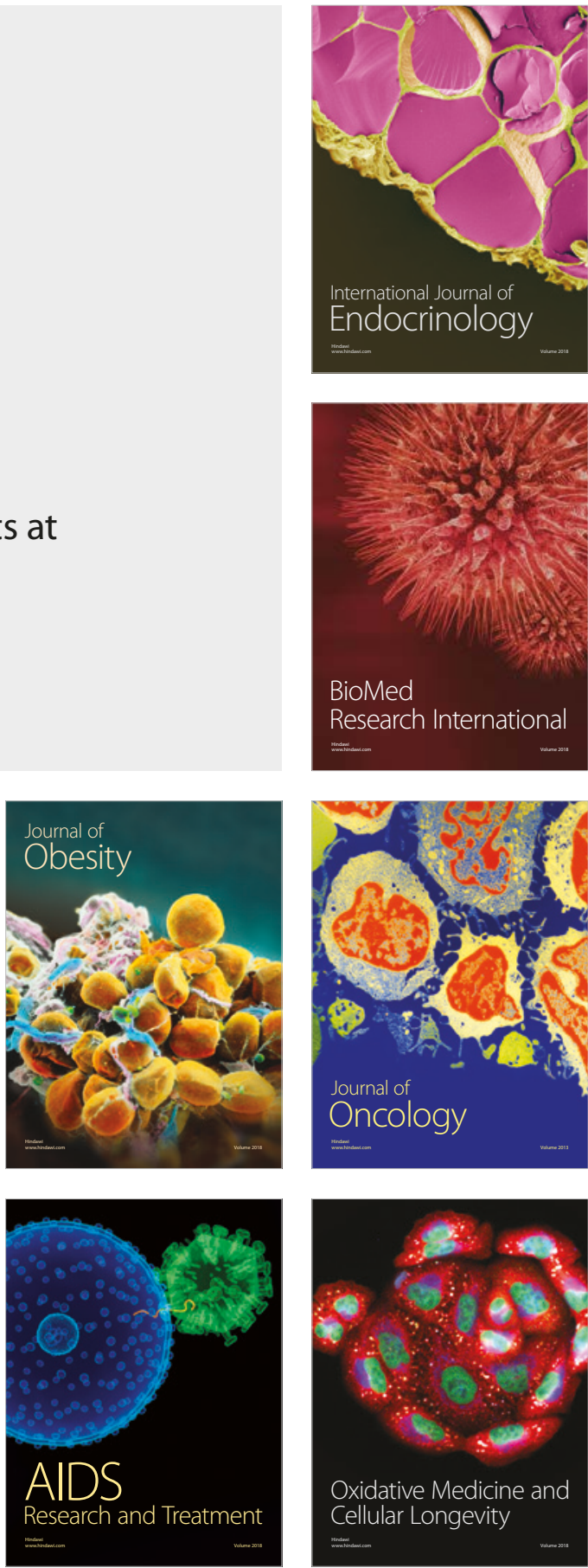